\title{
Biomonitoring: A Useful Tool for Occupational Health Practitioners
}

\author{
Nancy B. Hopf \\ Center for Primary Care and Public Health (Unisanté), Lausanne, Switzerland
}

"Excuse me. I did not capture the research area you mentioned. Could you please repeat?" said the woman in charge of registering new faculty members at the University of Lausanne, Switzerland, whom I had on the phone. This was about a decade ago. "Occupational hygiene specializing in biomonitoring" I repeated. Well, she informed me that this field was not listed as an option for research areas at the School of Biology and Medicine. "What about occupational hygiene?" I asked (I had been informed that in Europe, we call industrial hygienists, occupational hygienists). Again, a negative answer.

"What about occupational health?" I asked. Surely, this long-standing discipline exists, I thought. I had moved from Cincinnati, Ohio, 2 years prior with a $\mathrm{PhD}$ from the Medical College and the Department of Occupational and Environmental Health at the University of Cincinnati (UC), and several years working at the National Institute of Occupational Safety and Health (NIOSH). In our Occupational Health class at UC, we had studied the groundbreaking work the legendary occupational medical physician, Alice Hamilton, had carried out in the early 1900s. Then about the rights of working people to have a safe and healthy workplace, which gave way for the New Deal in the 1930s [1] that profoundly increased the role of the US federal government in Occupa- tional Safety and Health. In the 1960s, Unions pushed for federal legislation that ultimately resulted in the passage of the Mine Safety and Health Act of 1969 and the Occupational Safety and Health Act of 1970 [1]. Occupational health experts began monitoring workers' chemical exposures by measuring the internal dose of a chemical of interest. Human biomonitoring became a new tool in this era for assessing population-wide exposures to hazardous chemicals [2]. It gave a more reliable estimate of total exposures such as lead concentrations in urine [3]. Later, human biomonitoring methods sought to measure a chemical's biotransformations in the body, its metabolites.

Biomonitoring research is exploding now, several centuries after the occupational physician Bernardino Ramazzini formed the field of occupational medicine during the 17th century in Italy. The birthplace of the father of Occupational Health was in Europe, where I now work. "No," said my university of Lausanne colleague, "these research fields are not listed either. Could occupational hygiene be listed as physics, chemistry, biology, medicine, or mathematics?" the university colleague asked. I opted for chemistry because, as a nonclinician, medicine was out, but where does biomonitoring belong? (c) 2021 The Author(s). Published by S. Karger AG, Basel on behalf of NOVA National School of Public Health

This is an Open Access article licensed under the Creative Commons Attribution-NonCommercial-4.0 International License (CC BY-NC) (http://www.karger.com/Services/OpenAccessLicense), applicable to the online version of the article only. Usage and distribution for commercial purposes requires written permission.
Correspondence to:

Nancy B. Hopf, nancy.hopf@unisante.ch 
Biomonitoring has various applications including as an exposure assessment tool, which is central to publichealth efforts confirming health effects of environmental exposures and validating public-health policies [4]. Population-based biomonitoring studies have identified new chemicals found in the environment and in human tissues, monitored changes in exposures, and established the distribution of exposures among the general population. Biomonitoring data can be used to measure chemical exposures absorbed into the body, but only in conjunction with data from other disciplines such as epidemiology, toxicology, and pharmacokinetic modeling can biomonitoring provide a measure of potential health risk [4].

In the USA, industrial hygienists use the biomonitoring tool to assess occupational exposures, especially for workers with skin contact with the chemical of interest, as air monitoring would be inadequate, as it accounts for the inhalation route of exposure, not the skin. The industrial hygienists collect the biological material such as urine and exhaled breath samples, and with the help of an occupational nurse, blood samples. The industrial hygienist must then choose an appropriate chemical analytical laboratory that can analyze the compound itself or its metabolite(s) referred to as exposure biomarkers. The chosen laboratory sends the biomonitoring results to the industrial hygienist who is in charge of developing the exposure reduction strategies. These strategies are usually developed with the workers who know their job tasks and, thus, what is possible to change, company management, and the occupational physician. This is not the workflow in Switzerland where occupational biomonitoring can only be performed in conjunction with a medical doctor. In Switzerland, as is the case for many European countries, biomonitoring is regulated as medical screening similar to other clinical biomarkers such as cholesterol levels and liver enzyme concentrations. This powerful biomonitoring tool is thus underused as it is often not used by occupational doctors in the USA and occupational hygienists in Europe [5].

I believe that a multidisciplinary approach, including occupational health practitioners from different fields, can manage biomonitoring challenges better, and there are several. The National Academy of Sciences published a book in 2004 describing several of these, which are still valid today. One major challenge is the pace at which biomonitoring data are being generated and whose relevance to human health is unclear in many cases. The National Health and Nutrition Examination Survey (NHANES) in the USA started in the 1960s to assess the health and nu- tritional status of adults and children tracking exposure changes over time. A nationally representative sample of about 5,000 individuals is collected every year and includes about 60 analytical compounds such as heavy metal volatile organic compounds, pesticides, hormones, viruses, and phthalates (https://www.cdc.gov/nchs/nhanes/ about_nhanes.htm) [6]. The European Union have an ongoing human biomonitoring program HBM4EU that started in 2017 and will end this year. This effort will continue under a new 7-year project, the European partnership for the assessment of risks from chemicals (PARC). Population-based biomonitoring data give quantifiable concentrations of chemicals in people's blood or urine, but it does not necessarily mean that it causes a health risk or disease. Public-health officials need to transform these surveys into appropriate public-health policy responses, which is a challenge in itself.

From the occupational point of view, these biomonitoring survey data show the widespread nonoccupational exposures of chemicals among the general population. This knowledge is useful when deriving health-based biological limit values (BLVs). The occupational health practitioner can use these values for evaluating potential health risks in a particular population. Since 1982, the American Conference of Governmental Industrial Hygienists (ACGIH) has published biological exposure indices (BEIs) developed by the BEI committee. In Europe, the German Commission for the Investigation of Health Hazards of Chemical Compounds in the Work Area, known as the MAK Commission, has been deriving biological tolerance values (BATs) since 1981. These are updated and new values are added every year. The French Agency for Food, Environmental and Occupational Health \& Safety (ANSES) derives their equivalent of a BLV called Indicateurs Biologiques d'Exposition (IBEs). Japan established a Society for Research of Biological Monitoring in 1986, to develop reliable analytical methods, establish reference values, and implement and promote biological monitoring [7]. At present, the definition of what is a health-based limit value, let alone deriving one, is not harmonized across countries and institutions; thus, the interpretation of the biomonitoring results will depend on the BLV definition. The problem is that different approaches will result in different values for the same substance leading to a different level of risk management for workers exposed to the same substance. The commonality for deriving BEIs, BATs, and IBEs is first to consider exposure-effect and/or exposure-response relationships between biological monitoring values and health effects, and if insufficient scientific data exist, then a rela-
Port J Public Health 2021;39:69-71 DOI: $10.1159 / 000520703$
Hopf 
tionship between biological monitoring values and the corresponding airborne concentrations is elucidated. Why some of us are so passionate about developing these BLVs is that biomonitoring provides earlier warning than air monitoring, especially if skin exposures are likely. We believe that a harmonized approach could yield more BLVs and thus protect workers and reduce occupational health diseases. We have therefore launched a project with the OECD Working Party of Exposure Assessment (WPEA) and Working Party on Hazard Assessment (WPHA) in 2019 to harmonize the BLV approach and develop a guidance document [8]. A multidisciplinary team from more than 38 institutions leads this project.

In recent years, I have learned to appreciate the multidisciplinary approach working along with occupational medical doctors, and observe how the workers are more inclined to participate in biomonitoring programs because they trust the medical community more than the company management. Occupational hygienists are better at interpreting the biomonitoring data with respect to job exposures and possible exposure control options, while occupational medical doctors and nurses are better trained in understanding the ethical implications. We can all do better in communicating the results to the workers. Researchers have reported multiple benefits when reporting the biomonitoring results back to the workers such as better retention and recruitment into the biomonitoring program, advancing environmental health literacy, empowering participants to take actions to reduce exposures, encouraging shifts in government and industry practices, and helping researchers discover sources of exposure through participant consultation [9]. The challenges were among several, adopting protocols for notification of high exposures to chemicals without health guidelines. Therefore, I believe biomonitoring belongs in all the sciences (chemistry, physics, biology, mathematics, and medicine). To increase the use of the biomonitoring tool, we could imagine that biomonitoring classes could be taught for an interdisciplinary group of scientists and medical students and that ethical considerations and communication skills should be emphasized. We have all experienced for the past almost 2 years a global biomonitoring effort for mitigating COVID-19 exposure and disease. What is your opinion of biomonitoring communications and ethical considerations?

\section{Disclosure Statement}

Nancy B. Hopf is employed by the Center for Primary Care and Public Health (Unisanté), Lausanne, in Switzerland and is a Privat Docent at the University of Lausanne. The editorial's contents, including any opinions and/or conclusions expressed, are those of the author alone and do not necessarily reflect Unisanté policy in Switzerland.

\section{References}

1 Rosner D, Markowitz G. A short history of occupational safety and health in the United States. Am J Public Health. 2020 May;110(5): $622-8$.

2 Creager AN. Human bodies as chemical sensors: a history of biomonitoring for environmental health and regulation. Stud Hist Philos Sci. 2018 Aug;70:70-81.

3 Kehoe RA, Thamann M, Cholak J. On the normal absorption and excretion of lead. I. Lead absorption and excretion in primitive life. J Ind Hyg. 1933;15:257-72.

4 National Research Council. Human biomonitoring for environmental chemicals [Inter- net]. Washington: National Academies Press; 2006 [cited 2021 Oct 29]. Available from: http://www.nap.edu/catalog/11700.

5 Viegas S, Zare Jeddi M, Hopf NB, Bessems J, Palmen N, Galea KS, et al. Biomonitoring as an underused exposure assessment tool in occupational safety and health context: challenges and way forward. IJERPH. 2020;17(16): 5884.

6 Tranfo G. The growing importance of the human biomonitoring of exposure. IJERPH. 2020;17(11):3934.

7 Hopf NB, Fustinoni S. Biological monitoring of exposure to industrial chemicals. In: Harris
R, editor. Patty's industrial hygiene. 2021. Available from: https://doi.org/10.1002/ 0471435139.hyg042.pub38.

8 OECD. Occupational biomonitoring [Internet]. Paris: OECD; 2021 [cited 2021 Oct 29]. Available from: https://www.oecd.org/env/ ehs/risk-assessment/occupational-biomonitoring.htm.

9 Ohayon JL, Cousins E, Brown P, MorelloFrosch R, Brody JG. Researcher and institutional review board perspectives on the benefits and challenges of reporting back biomonitoring and environmental exposure results. Environ Res. 2017 Feb;153:140-9. 